\title{
Analysis of Bone Utilizing Infrared and Raman Chemical Imaging
}

\author{
Thomas J. Tague Jr. , Christian P. Schultz*, and Lisa Miller** \\ * Bruker Optics, Inc., 19 Fortune Drive, Billerica, MA 01821 \\ ** National Synchrotron Light Source, 725D, Brookhaven National Laboratory, Upton, NY $11973-$ \\ 5000
}

Osteoporosis is a disease that affects about 1 in 4 women over the age of 50 and about 1 in 8 of the male population over 50. Treatment regimens have been shown to be effective in slowing bone loss and in some cases yield an increase in bone density. In investigating the remodeling process, it is vital to be able to identify areas of new growth to evaluate the effectiveness of treatment regimens.

Contrast enhanced microscopy and fluorescence illumination have been employed to visualize and characterize monkey bone tibia tissue after necropsy. While detail in the bone structure is evident under brightfield and more so under darkfield illumination, distinction between older tissue and newly modeled bone is not possible. Newly modeled bone is evident using fluorescence illumination, where fluorescent markers have been used to facilitate clear observation of new tissue.

Direct chemical analysis of the mineral content in bone has been subsequently evaluated using infrared and Raman imaging. Higher concentrations of phosphate and carbonate are good indicators of an increase in bone mass production and exhibit good signatures in the infrared. The Raman scattering efficiency is also good for these molecules. The presence of fluorescence markers preclude the use of green excitation, but red excitation at $785 \mathrm{~nm}$ yielded excellent Raman results.

The ability to correlate the visual micrographs with infrared and Raman analysis provided a useful method for evaluating bone tissue. It was possible to identify newly modeled bone by the fluorescent markers and characterize changes induced by therapeutic drugs by infrared and Raman imaging. Traditionally, single element infrared detector based systems, which employ remote masks, have been used for this type of analysis. Evaluating fine structure such as newly modeled bone by infrared is challenging because the areas of interest approach the diffraction limit. When the area of interest is on the same order as the wavelength of light, diffraction limits the performance of these systems. Focal plane array based infrared imaging systems allow for much better signal to noise in approaching the diffraction limit and subsequently provide an excellent tool for evaluating fine tissue structure. The Raman chemical imaging analysis proved equally useful in chemically evaluating the bone mineral content. The Raman excitation laser wavelength used was in the visible, which yielded a smaller spatial resolution.

\section{References}

(1) L. M. Miller, et al., "Examination of Bone Chemical Composition in Osteoporosis Using Fluorescence - Assisted Synchrotron IR Microscopy”, Cellular Biology, in press.

(2) R. Mendelson, et al., "Infrared Spectroscopy, Microscopy, and Microscopic Imaging of Mineralizing Tissues. Spectra- Structure Correlations from Human Iliac Crest Biopsies. Journal of Biomedical Optics, 4 14-21 (1999). 
Fig1. Brightfield image of monkey bone tibia (left), the corresponding global infrared chemical image (right), and the infrared spectra showing the $\mathrm{CO}_{3}^{-2}$ band of interest (bottom).
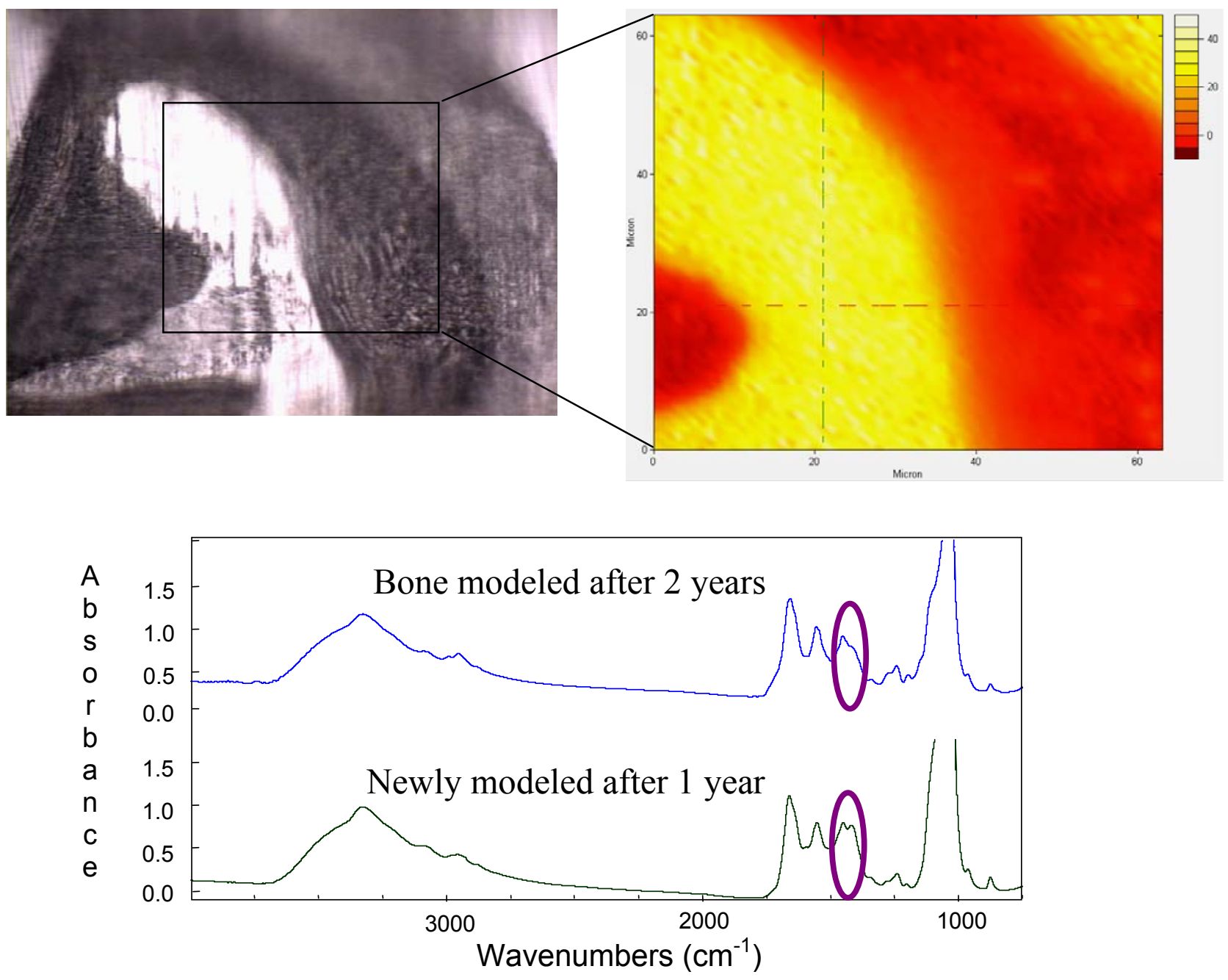

Fig 2. Brightfield image of monkey bone tibia (left) and corresponding Raman image (right)
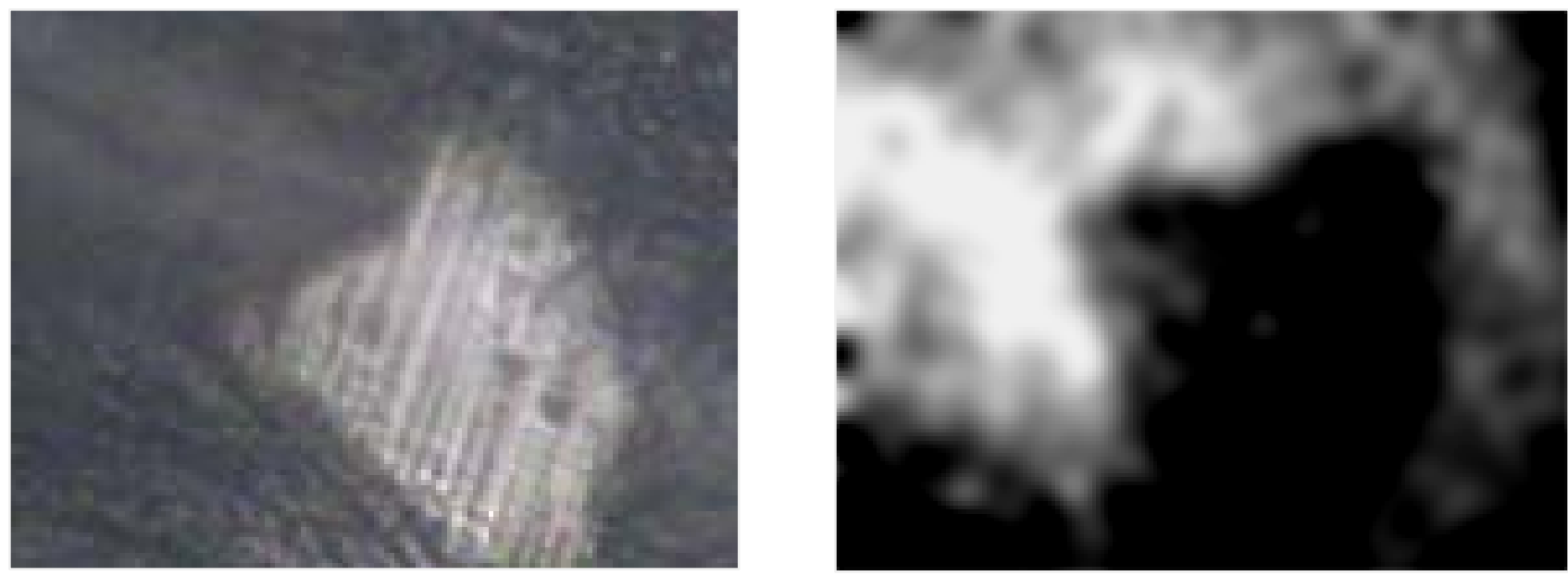